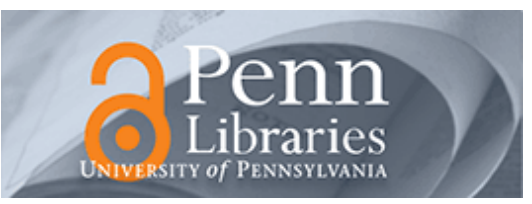

University of Pennsylvania ScholarlyCommons

December 2008

\title{
Temperature-Sensitive Nanocapsules for Controlled Drug Release Caused by Magnetically Triggered Structural Disruption
}

Ting-Yu Liu

National Chiao Tung University

Kun-Ho Liu

National Chiao Tung University

Dean-Mo Liu

National Chiao Tung University

San-Yuan Chen

National Chiao Tung University

I-Wei Chen

University of Pennsylvania, iweichen@seas.upenn.edu

Follow this and additional works at: https://repository.upenn.edu/mse_papers

\section{Recommended Citation}

Liu, T., Liu, K., Liu, D., Chen, S., \& Chen, I. (2008). Temperature-Sensitive Nanocapsules for Controlled Drug Release Caused by Magnetically Triggered Structural Disruption. Retrieved from

https://repository.upenn.edu/mse_papers/160

Pre-print version. To be published in Advanced Functional Materials, 2009. Publisher URL: http://dx.doi.org/ 10.1002/adfm.200801304

This paper is posted at ScholarlyCommons. https://repository.upenn.edu/mse_papers/160

For more information, please contact repository@pobox.upenn.edu. 


\title{
Temperature-Sensitive Nanocapsules for Controlled Drug Release Caused by Magnetically Triggered Structural Disruption
}

\author{
Abstract \\ Self-assembled nanocapsules containing a hydrophilic core and a crosslinked yet thermosensitive shell \\ have been successfully prepared using poly(ethylene-oxide)-poly(propylene-oxide)-poly(ethylene-oxide) \\ block copolymers, 4-nitrophenyl chloroformate, gelatin, and 1-ethyl-3-(3- dimethylaminopropyl) \\ carbodiimide. The core is further rendered magnetic by incorporating iron oxide nanoparticles via internal \\ precipitation to enable externally controlled actuation under magnetic induction. The spherical \\ nanocapsules exhibit a hydrophilic-to-hydrophobic transition at a characteristic but tunable temperature \\ reaching $40^{\circ} \mathrm{C}$, triggering a size contraction and shrinkage of the core. The core content experiences very \\ little leakage at $25^{\circ} \mathrm{C}$, has a half life about $5 \mathrm{~h}$ at $45^{\circ} \mathrm{C}$, but bursts out within a few minutes under \\ magnetic heating due to iron oxide coarsening and core/shell disruption. Such burst-like response may be \\ utilized for controlled drug release as illustrated here using a model drug Vitamin B12.
}

\section{Comments}

Pre-print version. To be published in Advanced Functional Materials, 2009. Publisher URL:

http://dx.doi.org/10.1002/adfm.200801304 


\section{FULL PAPER}

Nanocapsules

T.-Y. Liu, K.-H. Liu, D.-M. Liu,

S.-Y. Chen, I.-W. Chen $1-8$

Temperature-Sensitive Nanocapsules for Controlled Drug Release Caused by Magnetically Triggered Structural Disruption

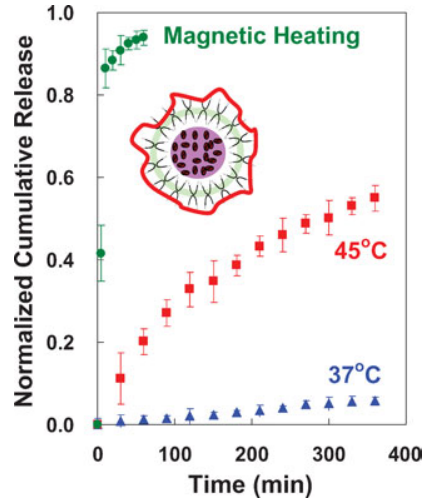

Nanocapsules containing magnetic iron oxide (brown precipitates) and a model drug (vitamin B12) encapsulated inside a cross-linked two-layered thermosensitive PEO-PPO-PEO shell abruptly shrink above the transition temperature $\left(40.5^{\circ} \mathrm{C}\right)$, which dramatically increases the drug release (compare two lower curves); they also release the drug in a burst upon remote magnetic heating (upper curve). 


\title{
Temperature-Sensitive Nanocapsules for Controlled Drug Release Caused by Magnetically Triggered Structural Disruption
}

\author{
By Ting-Yu Liu, Kun-Ho Liu, Dean-Mo Liu, San-Yuan Chen, and I-Wei Chen*
}

Self-assembled nanocapsules containing a hydrophilic core and a crosslinked yet thermosensitive shell have been successfully prepared using poly(ethylene-oxide)-poly(propylene-oxide)-poly(ethylene-oxide) block copolymers, 4-nitrophenyl chloroformate, gelatin, and 1-ethyl-3-(3dimethylaminopropyl) carbodiimide. The core is further rendered magnetic by incorporating iron oxide nanoparticles via internal precipitation to enable externally controlled actuation under magnetic induction. The spherical nanocapsules exhibit a hydrophilic-to-hydrophobic transition at a characteristic but tunable temperature reaching $40^{\circ} \mathrm{C}$, triggering a size contraction and shrinkage of the core. The core content experiences very little leakage at $25^{\circ} \mathrm{C}$, has a half life about $5 \mathrm{~h}$ at $45^{\circ} \mathrm{C}$, but bursts out within a few minutes under magnetic heating due to iron oxide coarsening and core/shell disruption. Such burst-like response may be utilized for controlled drug release as illustrated here using a model drug Vitamin B12. will require a careful design of material constituents and their self-assembly. Here we undertake such a study using poly (ethylene-oxide)-poly(propylene-oxide)-poly (ethylene-oxide) (PEO-PPO-PEO) copolymers and iron oxide nanoparticles as building blocks for the magnetic nanoparticle and vitamin B12 as a model drug; crosslinking agents were also introduced to modify the composite nanostructure.

As background, the idea of using external magnetic fields to achieve drug release from polymer composites was first reported by Kost et al. who demonstrated insulin release from a magnetic composite of ethylene vinyl acetate under a low frequency magnetic field. ${ }^{[1-2]}$ Recently, De Paoli et al. $^{[3]}$ reported magnetically enhanced dextran release (which simulates protein release) from a magnetic nanocomposite made of a collagen gel. Magnetic biocompatible iron oxide nanoparticles were used in the latter study as in our previous work on ferrogels, ${ }^{[4]}$ but they both involved bulk gels instead of colloids. Colloids of iron oxide nanoparticles can be used as a delivery vehicle: for example, a single-strand DNA can be grafted to the nanoparticle; a dye-labeled complement can then be reversibly associated to or dissociated from it depending on the temperature. ${ }^{[5]}$ On thermally sensitive polymers, Choi et al. ${ }^{[6]}$ reported pluronic/heparin nanocapsules that exhibited a reversible $(1000 \times)$ volume transition when cycled between 25 and $37^{\circ} \mathrm{C}$. Their study built on the well-known properties of PEO-PPO-PEO triblock polymers (biocompatible and commercially known as pluronic) that manifest a range of critical micellization temperature (CMT) for volume/hydrophobicity transition, but it further enhanced the volume change by crosslinking the outer shell. To aim at an unprecedentedly fast drug release under a remote magnetic trigger, we have combined these considerations in our design which contains (i) a collapsible magnetic core of iron oxide immersed in a water solution of vitamin B12, (ii) a fast-breathing nanosized two-layer shell of thermally responsive PEO-PPO-PEO polymer, and (iii) a crosslinked outer shell that stabilizes the nanoshell while maintaining the CMT, the volume change and the drug release. The preparation procedure following the selfassembly schemes of Figure $1 \mathrm{a}$ and $\mathrm{b}$, the experimental evidence (from transmission electron microscopy, dynamic light scattering) in support of the Schemes, and the thermal responses and drug release characteristics of the nanoparticles are described below.
DOI: 10.1002/adfm.200801304

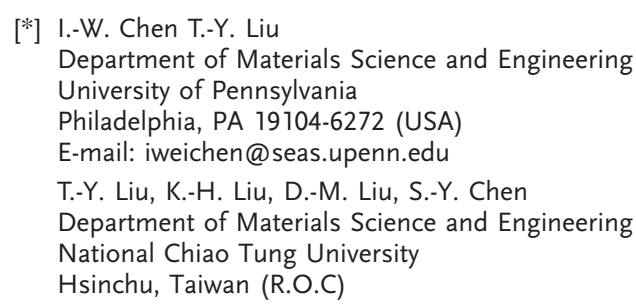

Department of Materials Science and Engineering

University of Pennsylvania

Philadelphia, PA 19104-6272 (USA)

E-mail: iweichen@seas.upenn.edu

T.-Y. Liu, K.-H. Liu, D.-M. Liu, S.-Y. Chen

Department of Materials Science and Engineering

National Chiao Tung University

Hsinchu, Taiwan (R.O.C)

Thermally sensitive drug delivery carriers that can be remotely actuated are attractive for therapeutic and patient management. One possible approach is to use a composite carrier made of a magnetic core inside a thermally sensitive polymer micelle with a temperature-dependent drug release profile, so that when the mexternal magnetic stimul idea is a natural extension of two concepts, (i) magnetic heating that has been used for tumor treatment (i.e., hyperthermia) and (ii) hydrophobicity transition that has compatibility, fast actuation, high on/off ratio of drug release and an operation temperature at about the physiological temperature

Ad. Funct. Mater. $2008,18,1-8$ 
a)
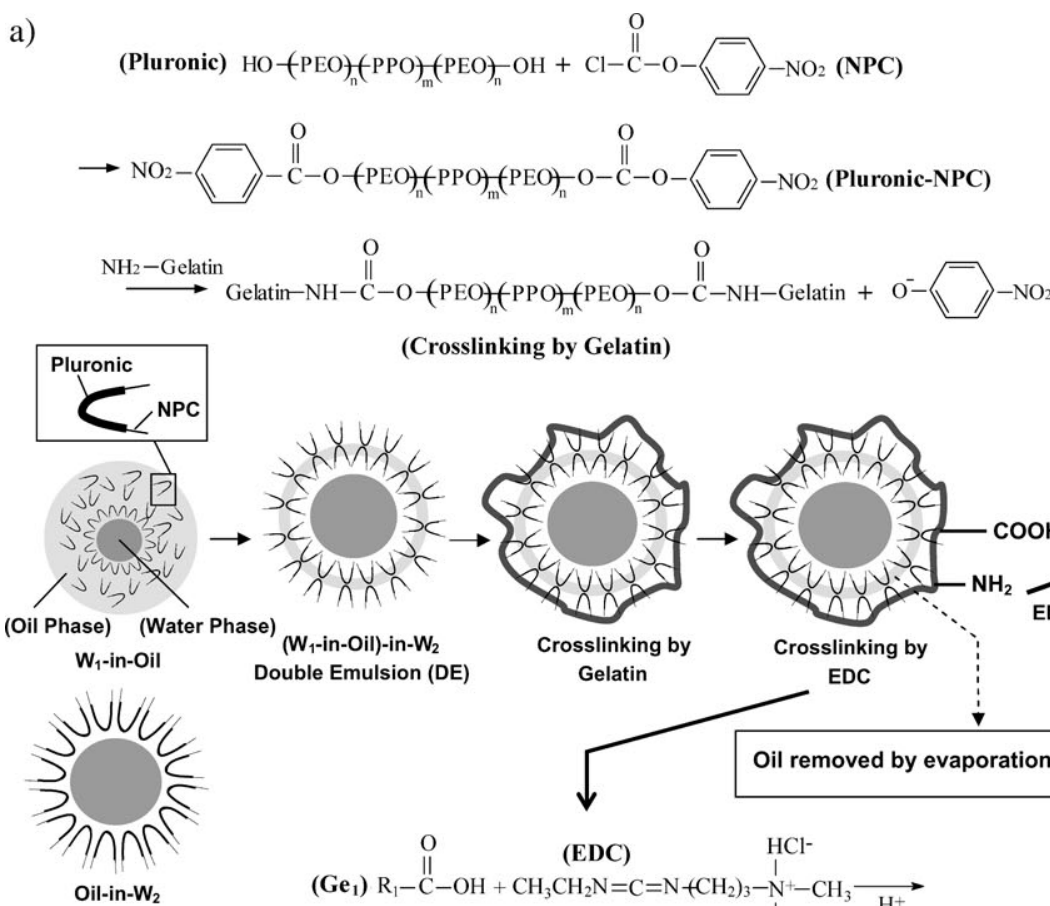

(Crosslinking by Gelatin)

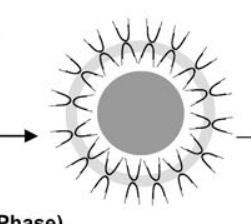

$\left(W_{1}\right.$-in-Oil)-in-W

Double Emulsion (DE)
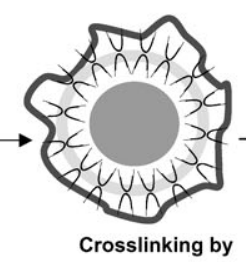$$
\text { Gelatin }
$$

Gelatin

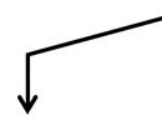

( EDC

Oil removed by evaporation

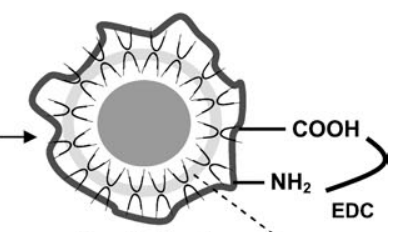
EDC

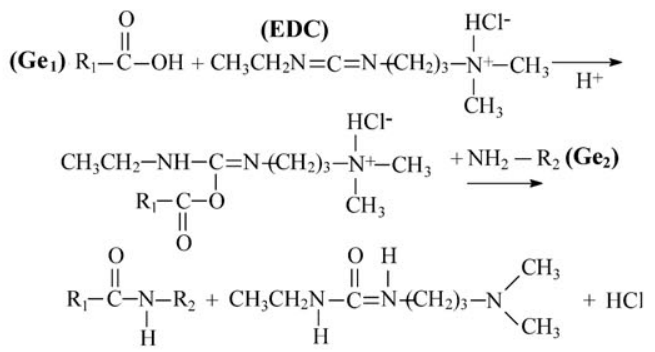

b)

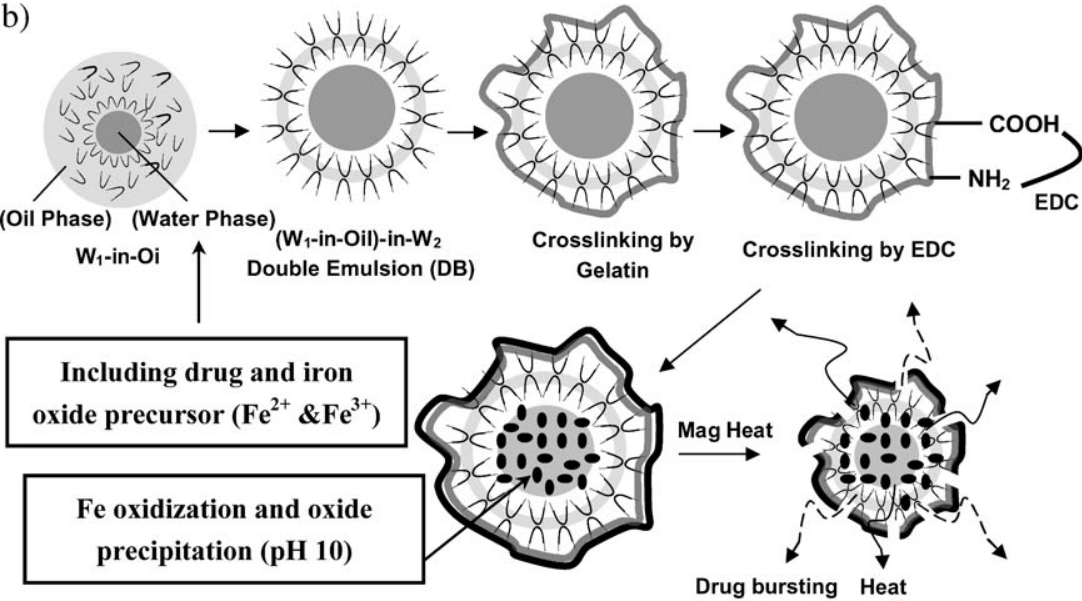

Core Collapse

Figure 1. a) Chemical reactions, self-assembly and schematic structures of various nanocapsules, b) procedure for encapsulating drug and iron oxide into nanocapsules and events triggered by magnetic heating: volume shrinkage, core collapse, heat conduction, and drug release.

NPC and F68-NPC in deuterated chloroform $\left(\mathrm{CDCl}_{3}\right)$ solution. The activated polymer differs from the unactivated one in that the terminal alcohols on PEO-PPO-PEO are converted into nitrophenyl groups in a chloraformate environment. This is supported by the spectra which give evidence of protons in the ortho (o)-position (near the $-\mathrm{NO}_{2}$ side) in the nitrophenyl $\left(\mathrm{NO}_{2} \mathrm{Ph}\right)$ group, protons in the meta $(m)$-position (near the $-\mathrm{COO}$ side) in the nitrophenyl group, and signals associated with $-\mathrm{NO}_{2} \mathrm{Ph}-\mathrm{OCOO}-\mathrm{CH}_{2}$ which identifies NPC's attachment to the $-\mathrm{OH}$ group of $\mathrm{PEO}$. Other structures indicate a PEO-PPO-PEO backbone including the $\mathrm{PEO}-\mathrm{CH}_{2}$-units and the $\mathrm{PPO}-\mathrm{CH}_{3}$ units.

\subsection{Size of Nanocapsules}

Double-layer F127-NPC and F68-NPC nanocapsules of different sizes at $25^{\circ} \mathrm{C}$ are shown in Figure 2. They are F127-NPC-DE in Figure 2a and F68-NPC-DE in Figure 2b, both in the form of double-emulsion ( $W_{1}$-in-oil-in$\mathrm{W}_{2}$ ), to be compared with single-emulsion (oilin- $\mathrm{W}_{2}$ ) nanocapsules (F127-NPC-SE and F68NPC-SE) and $\mathrm{W}_{1}$-in-oil inverse micelles (F127NPC-W1-in-oil and F68-NPC-W1-in-oil in the insets). For both polymers, the size of the nanocapsules decreases with increasing content of the polymer, but the size is always smaller for the F127-series than for the F68series. Regardless of the polymer used, the resulting nanocapsules normally show a size decrease in the order of $\mathrm{DE}>\mathrm{SE}>\mathrm{W}_{1}$-in-oil. Moreover, in the case of the DE and SE series, a constant size is reached when the polymer concentration is around $1.0 \mathrm{~g} \mathrm{~mL}^{-1}$. This critical concentration for maintaining a constant size was employed in all subsequent experiments.

\subsection{Size Variation of the Crosslinked Nanocapsules}

The size distributions of double-layer nanocapsules F127-NPC-DE before and after gelatin-cross-linking are shown in Figure 3. With the addition of $1 \mathrm{mg} \mathrm{mL}^{-1}$ gelatin in the $\mathrm{W}_{2}$ phosphate buffered solution (PBS) solu-

\section{Results}

\section{2.1. Structure of NPC-Activated F127 and F68}

$3 \quad{ }^{1}$ H NMR spectra are shown in the Supporting Information Figure

$4 \mathrm{~S} 1 \square^{\mathrm{Q} 1}$ Please check the change made here in the text. $\square$ for F127tion, the size increases slightly from a peak value of $28.4-35.6 \mathrm{~nm}$. This increment is mostly caused by a small increase in the CMT (from 21.9 to $22.8^{\circ} \mathrm{C}$, see Table 1 ) due to the presence of gelatin; this point will be supported by other CMT data to be detailed later. A high concentration of gelatin, e.g., 5 and $10 \mathrm{mg} \mathrm{ml}^{-1}$, broadens the size distribution considerably and causes a secondary peak at 9 

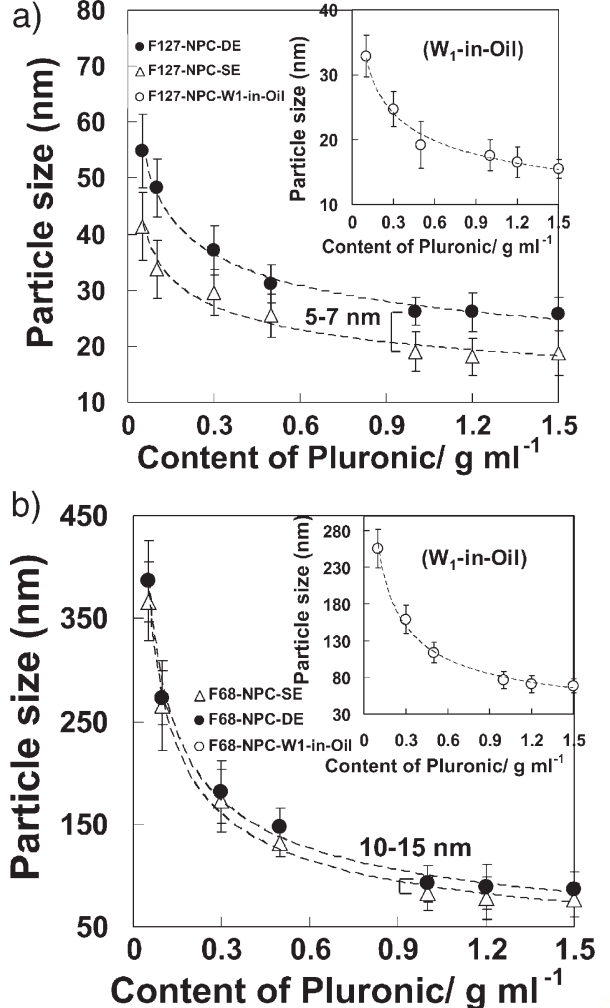

Figure 2. Diameter of nanocapsules measured by DLS of a) F127 and b) F68 series. DE is a water-in-oil-in-water structure, SE is an oil-in-water structure, and the inset is a water-in-oil structure.

a very large size (Fig. 3, inset), indicating agglomeration has occurred possibly by forming cross-linking bridges between nanocapsules. Accordingly, an optimal concentration of $1 \mathrm{mg}$ $\mathrm{ml}^{-1}$ gelatin was selected in the $\mathrm{W}_{2}$ PBS solution for further study.

Likewise, the size of nanocapsules after the second crosslinking using EDC was measured where a size decrease from 35.6 to $30.5 \mathrm{~nm}$ was found as shown in Table $1\left(d_{\mathrm{RT}}\right)$. A similar trend

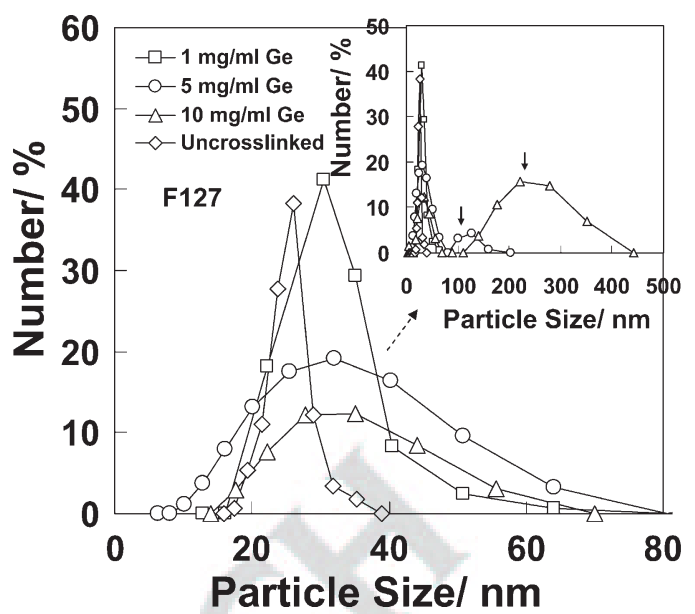

Figure 3. Diameter distribution of F127-NPC nanocapsules after crosslinking by different amount of gelatins. Inset: same with a more extended size range.

was found for F68 nanocapsules (Table 1). Since the change in 1 CMT due to EDC, from 22.8 to $22.4{ }^{\circ} \mathrm{C}$ (Table 1 ) is relatively small, 2 the size shrinkage may be attributed to a more compact structure 3 after $-\mathrm{NH}_{2}$ and $-\mathrm{COOH}$ cross-linking. 4

A freshly formed F68-NPC-DE nanocapsule after double 5 emulsion has a spherical geometry as shown in the TEM image in 6 Figure 4a. After washing five times and dialysis, the nanocapsule 7 (Fig. 4b) is about $100 \mathrm{~nm}$, which is very close to the DLS peak 8 value (see Table 1). After gelatin and EDC cross-linking there is 9 little further change in size but a higher electron density has 10 developed on the particle skin (Fig. 4c).

\subsection{Precipitation and Encapsulation of Iron oxide}

Evidence for the formation of iron oxide (IO) nanocrystals inside the nanocapsules is shown in Figure 5 for F68-NPC-DE after gelatin and EDC crosslinking. The size is again about $100 \mathrm{~nm}$

Table 1. Characteristics of $\mathrm{F} 127$ and F68-series nanocapsules at $1 \mathrm{~g} \mathrm{~mL}^{-1}$ pluronic.

\begin{tabular}{|c|c|c|c|c|c|c|c|c|c|}
\hline \multirow[t]{2}{*}{ Samples } & \multicolumn{2}{|c|}{ Non-activated } & \multicolumn{2}{|c|}{ NPC-activated } & \multicolumn{2}{|c|}{ Gel-modified } & \multicolumn{2}{|c|}{ EDC-modified } & \multirow{2}{*}{$\begin{array}{c}\text { Iron oxide } \\
\text { F68-IO }\end{array}$} \\
\hline & $\mathrm{F} 127$ & F68 & F127-NPC & F68-NPC & F127-Ge & F68-Ge & F127-EDC & F68-EDC & \\
\hline $\mathrm{CMT}\left[{ }^{\circ} \mathrm{C}\right]$ & 25.8 & 43.2 & 21.9 & 39.3 & 22.8 & 40.1 & 22.4 & 39.6 & 40.5 \\
\hline \multicolumn{10}{|c|}{ Size and size ratio } \\
\hline$d_{\max }[\mathrm{nm}][\mathrm{a}]$ & 115.7 & 143.1 & 78.2 & 86.4 & 91.4 & 105.3 & 82.1 & 97.0 & 113.1 \\
\hline$d_{\mathrm{RT}}[\mathrm{nm}][\mathrm{b}]$ & 60.8 & 135.2 & 28.4 & 85.3 & 35.6 & 101.5 & 30.5 & 94.2 & 108.3 \\
\hline$d_{\min }[n m][c]$ & 23.6 & 22.1 & 27.3 & 22.0 & 27.3 & 23.8 & 25.1 & 23.6 & 43.1 \\
\hline$d_{\max } / d_{\min }$ & 4.9 & 6.5 & $-\bar{\equiv}$ & $-\bar{\equiv}$ & 3.3 & 4.4 & 3.2 & 4.1 & 2.6 \\
\hline \multicolumn{10}{|c|}{ Drug release (ratio) } \\
\hline Ratio $_{45 / 4}[\mathrm{~d}]$ & - & 12.1 & - & - & - & 39.4 & - & 45.5 & 57.3 \\
\hline Ratio $_{45 / 25}[\mathrm{e}]$ & - & 5.9 & - & - & - & 11.6 & - & 14.3 & 17.2 \\
\hline Ratio $_{45 / 37}[\mathrm{f}]$ & - & 3.5 & - & - & - & 6.8 & - & 8.2 & 9.5 \\
\hline
\end{tabular}

[a] $10^{\circ} \mathrm{C}$ for F 127 -series and $20^{\circ} \mathrm{C}$ for F68-series. [b] Room temperature $\left(25^{\circ} \mathrm{C}\right)$. [c] $40^{\circ} \mathrm{C}$ for F 127 -series and $50{ }^{\circ} \mathrm{C}$ for F68-series. [d] Ratio of cumulative percentage release after $6 \mathrm{~h}$ at $45^{\circ} \mathrm{C}$, to that at $4{ }^{\circ} \mathrm{C}$. [e] Ratio of cumulative percentage release after $6 \mathrm{~h}$ at $45^{\circ} \mathrm{C}$, to that at $25{ }^{\circ} \mathrm{C}$. [f] Ratio of cumulative percentage release after $6 \mathrm{~h}$ at $45^{\circ} \mathrm{C}$, to that at $37^{\circ} \mathrm{C}$. 

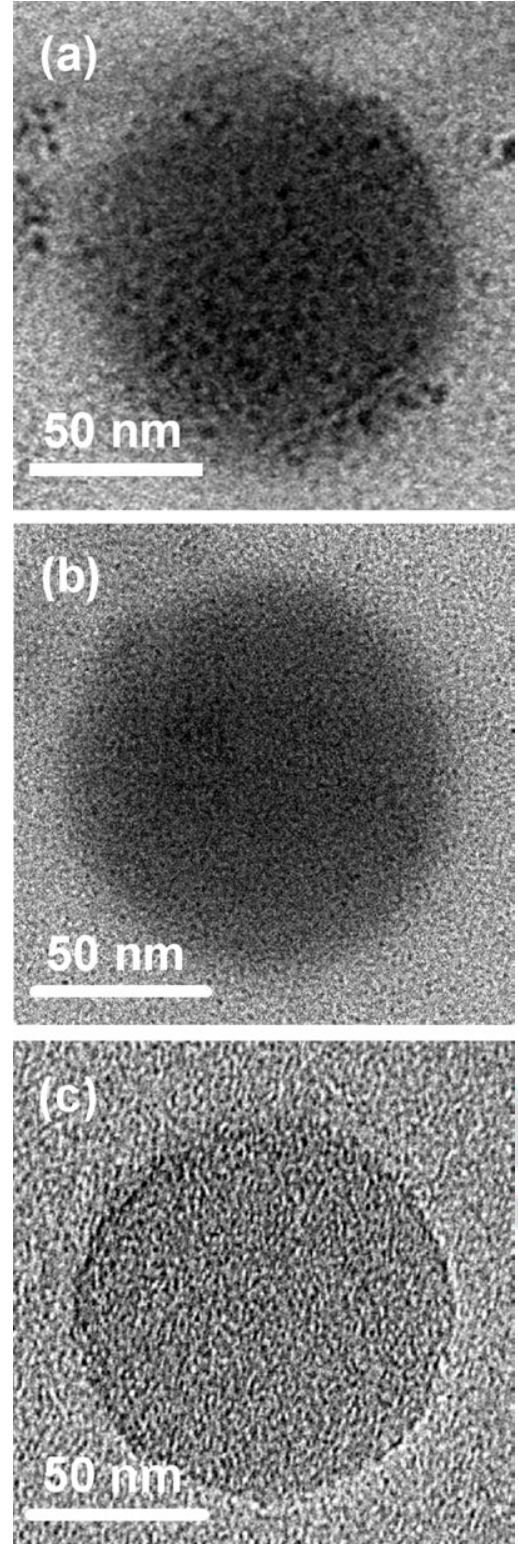

Figure 4. Transmission electron micrographs of F68 series nanocapsules a) before dialysis, b) after repeated washing and dialysis, and c) after gelatin and EDC cross-linking.

1 (Fig. 5a) and the skin $(6-8 \mathrm{~nm})$ is clearly of a higher electron 2 density (Fig. 5b). In addition, there is evidence of chain crystallization (periodic fringes in highlighted boxes in Fig. 5b) 4 in the corona of the nanocapsules. At higher magnification, the 5 nanocapsule shows a contrast modulation due to particles of a 6 size of $5-10 \mathrm{~nm}$ (Fig. 5c). These particles are crystalline as 7 evidenced by the selected area diffraction pattern in Figure 5d, 8 indicating the $\mathrm{Fe}_{3} \mathrm{O}_{4}$ crystal planes of [200], [311], [400], and [511].

\subsection{Thermosensitive Behavior and CMT}

10

11 Nanocapsules exhibit thermally sensitive behavior similar to that of the PEO-PPO-PEO polymer showing shrinkage above the
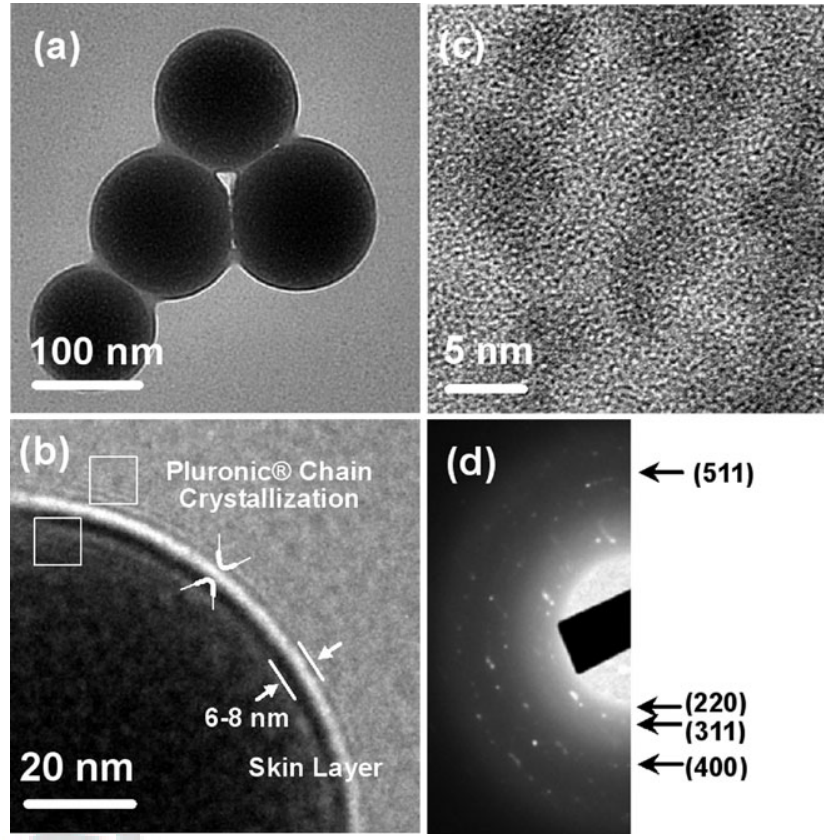

Figure 5. Transmission electron micrographs of a) fully cross-linked F68 nanocapsules containing iron oxide nanoparticles, b) a high electron density shell of a thickness about $6-8 \mathrm{~nm}$, with a corona region highlighted to show periodic modulation presumably due to polymer chain crystallization, and c) contrast modulation due to iron oxide nanoparticles. d) Selected area electron (SAE) diffraction pattern of several single crystals of various orientations.

CMT, signifying a hydrophilic/hydrophobic transition. The CMT and volume change can be altered by the chemical modification (e.g., NPC and gelatin) which provides a way for fine tuning to suit the drug release application. This was determined by measuring the capsule size as a function of temperature, shown in Figure 6, where a transition is manifested by a large volume shrinkage. For data quantification, the CMT is defined as the inflection point of the curve, and a size ratio is defined using $d_{\max } / d_{\min }$ where $d_{\max }$ is

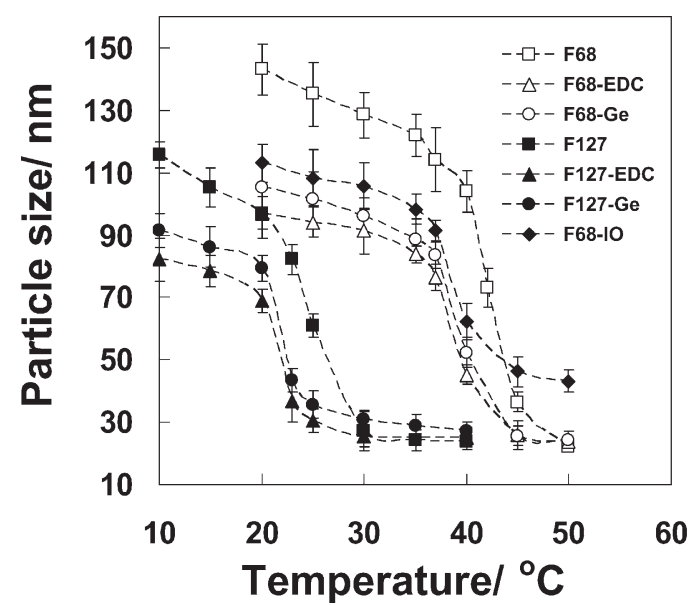

Figure 6. Diameter of nanocapsules measured by DLS decreases abruptly at about CMT. F68 series has higher CMT than F127 series. 
1 the maximum particle diameter below the CMT at the lowest 2 measurement temperature and $d_{\min }$ is the minimum particle 3 diameter above the CMT at the highest measurement temperature. It is then clear that the larger size of the F68 series is mainly due to a much higher CMT $\left(43.2^{\circ} \mathrm{C}\right)$ than that of F127 $\left(25.8^{\circ} \mathrm{C}\right)$. 6 (This largely reflects a higher $\mathrm{EO} / \mathrm{PO}$ ratio in $\mathrm{F} 68$, which is 5.24, compared to that of F127, which is 3.08.) In addition, there is a slight decrease (about $4{ }^{\circ} \mathrm{C}$ ) of CMT for activated polymers, 9 presumably because NPC addition causes an increase of 0 hydrophobicity. In contrast, the increase (about $1{ }^{\circ} \mathrm{C}$ ) in CMT 1 is small in gelatin-cross-linked nanocapsules, and the increase 2 (about $0.5^{\circ} \mathrm{C}$ ) when EDC was added is even smaller. These 3 changes of CMT summarized in Table 1 are consistent with the 4 size data at $25^{\circ} \mathrm{C}$.

The size ratio of the nanocapsule significantly decreases when it is cross-linked by gelatin, but the further decrease is small when it is additionally cross-linked by EDC (row 5 in Table 1). 8 Incorporating IO into F68-EDC decreases the size ratio from 4.1 9 (corresponding to a volume ratio of about 67) to 2.6 20 (corresponding to a volume ratio of 18); see row 6 in Table 1. 21 From Figure 6, it is clear that the IO addition causes a decrease of $2 d_{\max }$ and an increase of $d_{\text {min }}$, so both the hydrophilic "swollen" 3 state and the hydrophobic "shrunk" state appear to be sterically 24 constrained from reaching their respective fully relaxed sizes. 25 Since the F68 series shows a higher ratio than the F127 series, it 6 was selected to encapsulate B12 for drug release studies.

\section{2.6. Drug Release with and without Magnetic Heating}

Table 2. Summary of fitting constants ( $n$ and $k$ ) for cumulative release, apparent diffusivity, and estimated half life $t_{50}$ reaching $50 \%$ cumulative release.

\begin{tabular}{lrlrrr}
\hline Sample & Temp. $\left[{ }^{\circ} \mathrm{C}\right]$ & \multicolumn{1}{c}{$n$} & $k \times 10^{3}$ & $D_{\mathrm{e}} \times 10^{9}\left[\mu \mathrm{m}^{2} \mathrm{~min}^{-1}\right]$ & $t_{50}[\mathrm{~h}]$ \\
\hline F68 & 4 & 0.781 & 0.62 & 0.96 & 41.3 \\
& 25 & 0.77 & 1.22 & 3.63 & 27.5 \\
& 37 & 0.762 & 2.00 & 8.49 & 16.3 \\
F68-Ge & 45 & 0.588 & 28.15 & 177.12 & 2.1 \\
& 4 & 0.799 & 0.10 & 0.03 & 270.6 \\
& 25 & 0.783 & 0.47 & 0.62 & 82.9 \\
& 37 & 0.773 & 0.79 & 1.73 & 42.1 \\
F68-EDC & 45 & 0.603 & 18.26 & 87.57 & 3.9 \\
& 4 & 0.808 & 0.07 & 0.02 & 277.8 \\
& 25 & 0.787 & 0.33 & 0.31 & 83.1 \\
& 37 & 0.776 & 0.59 & 0.91 & 41.5 \\
F68-EDC-IO & 45 & 0.616 & 14.18 & 60.67 & 6.0 \\
& 4 & 0.846 & 0.05 & 0.02 & 416.7 \\
& 25 & 0.84 & 0.24 & 0.25 & 92.3 \\
& 37 & 0.833 & 0.41 & 0.85 & 41.6 \\
& 45 & 0.615 & 15.71 & 73.02 & 4.8 \\
\hline
\end{tabular}

(neat polymer), F68-Ge (gelatin cross-linked), F68-EDC (further 1 EDC crosslinked), and F68-IO (IO-containing and fully cross- 2 linked). The data were obtained at four temperatures, 4, 25, 37, 3 and $45^{\circ} \mathrm{C}$, and fitted with Equation (2) Equation 1 has been 4 changed to Equation 2 and vice versa in order to make it in 5 sequence. Please check. giving the fitting parameters listed in 6 Table 2. Although some data (those with $n$ far away from 0.5 ) do 7 not warrant an interpretation based on diffusion mechanisms, to 8 allow a more quantitative comparison we nevertheless force-fit 9 the data with the following expression ${ }^{[7]}$ to obtain the apparent 10 "diffusivity" $D_{\mathrm{e}}$ listed in Table 2,

$\frac{M_{t}}{M_{\infty}}=4\left(\frac{D_{\mathrm{e}} t}{\pi d^{2}}\right)^{1 / 2}$

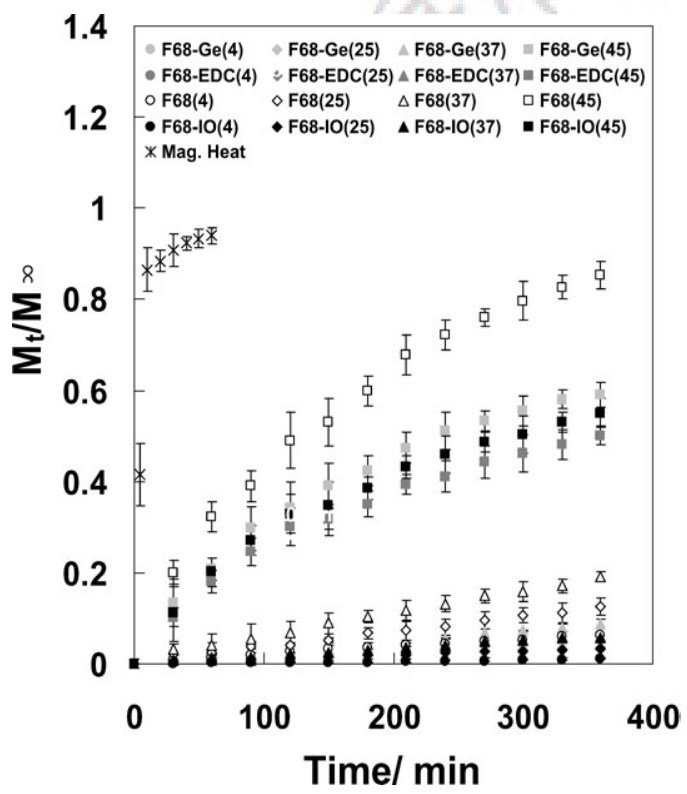

Figure 7. Cumulative vitamin $\mathrm{B} 12$ release as a function of time for four F68 series nanocapsules at various temperatures from 4 to $45^{\circ} \mathrm{C}$ as indicated in the parentheses. Many low temperature data are too close to be resolved on this graph but their relative order is the same as $45^{\circ} \mathrm{C}$. Release data under an external magnetic field for F68-IO also shown (mag heat).
This fitting was performed for $M_{t} / M_{\infty}<0.6$. It is then clear 12 that there is a systematic reduction of $D_{\mathrm{e}}$ in the order of 15 F68 $>$ F68-Ge $>$ F68-EDC $\sim$ F68-EDC-IO, in addition to the 16 obvious increase of $D_{\mathrm{e}}$ with the temperature. This indicates that 17 vitamin B12 release is a thermally activated process, and 18 crosslinking of the shell slows down the release.

From a practical viewpoint, a large on/off ratio is desired. This 20 would require a very large ratio of the $45^{\circ} \mathrm{C}$ release to that at either 21 the physiological temperature $\left(37^{\circ} \mathrm{C}\right)$ or the storage/application 22 temperature $\left(4^{\circ} \mathrm{C} / 25^{\circ} \mathrm{C}\right)$. These ratios are listed in Table 1 using 23 the cumulative release after $6 \mathrm{~h}$. It is clear that crosslinking 24 significantly increases the ratio, and the incorporation of IO does 25 not cause any adverse effect. The ratio comparing $45^{\circ} \mathrm{C}(>\mathrm{CMT}) \quad 26$ and $37^{\circ} \mathrm{C}(<\mathrm{CMT})$ is 9.5 indicating a clear effect of the transition. 27 Meanwhile, the slow release at 4 and $25^{\circ} \mathrm{C}$ being only $1 / 57$ and $1 / 28$ 17 , respectively of that at $45^{\circ} \mathrm{C}$ assures a long shelf time of this 29 drug carrier.

Lastly, the effect of magnetic heating (with the solution held in 31 a water bath of $15^{\circ} \mathrm{C}$ ) is shown in Figure 7 (see symbols $\times$ What 32 symbol???ם) which evidently has a much larger cumulative 33 release in just a few minutes: about $40 \%$ cumulative release is 34 reached in the first $5 \mathrm{~min}$, which is about 20 times the rate 35 

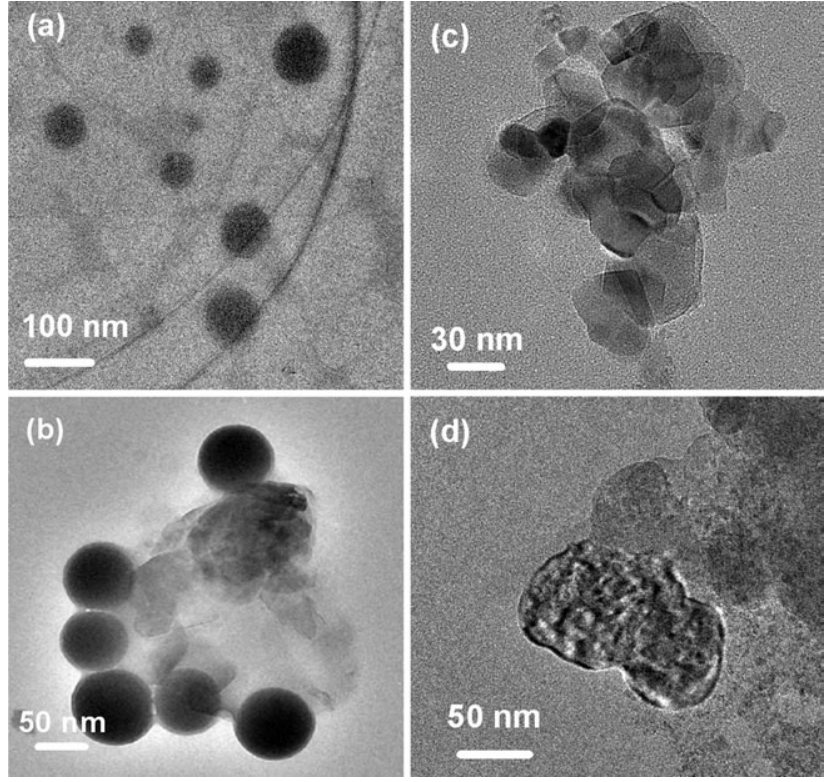

Figure 8. Transmission electron micrographs of F68-1O nanocapsules showing a) uneven shrinkage after heating to $45^{\circ} \mathrm{C}$; b) unevenly restructured core and shell after magnetic heating, with c) structure disruption and faceting, and d) crystal coarsening.

1 normally achieved at $45^{\circ} \mathrm{C}$. According to Figure 6 the shrinkage is 2 already nearly complete at $45^{\circ} \mathrm{C}$, so magnetic heating should not 3 cause much more squeezing of the core. Therefore, the burst-like 4 accelerated release under magnetic heating cannot be attributed 5 to the volume change alone.

The TEM micrograph presented in Figure 8a shows that when nanocapsules experienced a thermal excursion to $45^{\circ} \mathrm{C}$ but without a magnetic field they remained spherical although some 9 (smaller spheres in Fig. 8a) had apparently shrunk indicating 0 drug release may have occurred. In contrast, after exposure to a 1 magnetic field many nanocapsules lost their spherical shape, as 2 shown in Figure 8b-d. Some appeared to be faceted suggesting 3 recrystalization (Fig. 8c); others have coarsened considerably 14 suggesting grain growth (Fig. 8d). Clearly, these changes are 15 highly disruptive which may correspond to the scenario depicted 16 in Figure $1 \mathrm{~b}$ leading to a burst of drug release.

\section{3. Discussion}

measurement of pluronic 105 (containing $(\mathrm{EO})_{37}(\mathrm{PO})_{56}\left(\mathrm{EO}_{37}\right) \quad 1$ blocks) gave a double-layer width of $9.5 \mathrm{~nm}$, which is of the same 2 order of magnitude. ${ }^{[8]}$

For comparison, we next estimate the single-layer thickness 4 using another method. From Figure 2, we see that there is a 5 critical concentration of $1 \mathrm{~g}$ pluronic/1 $\mathrm{mL}$ oil above which a 6 constant size is reached for both types (F127 and F68) of 7 nanocapsules. This suggests that the excess pluronic is dissolved 8 and not incorporated in the shell. Since the density of pluronic and oil is similar, the above critical concentration corresponds to a 10 volume ratio $V_{\text {pluronic }} / V_{\text {oil }}$ about 1 for the "equilibrated" oil-in- 11 water micelles of single-shell. The single-shell thickness of such equilibrated micelle can be estimated from the size data in Figure 2 and the spherical geometry with a core radius $r$ and a shell thickness $a$ : the shell to (oil) core volume ratio is about $r / 3 a$ and the diameter $2 r+2 a$ should correspond to the steady-state size in Figure 2 (the single-emulsion nanocapsules). From this, the single-shell thickness is estimated to be about $2.4 \mathrm{~nm}$ for F127 and $10.3 \mathrm{~nm}$ for $\mathrm{F} 68$, at $25^{\circ} \mathrm{C}$. These values fall in the same range as estimated before.

\subsection{Stability of Nanocapsules and Controlled Drug Release}

Nanocapsules reported in this work are highly stable at room temperature and below. This is reflected in the low release rate at 4 and $25^{\circ} \mathrm{C}$. An estimate of the half life $\left(t_{50}\right)$ required to reach $50 \%$ release may be obtained by replotting Figure 7 in a log-log form to extrapolate it to $M_{t} / M_{\infty}=0.5$ (figure not shown). ${ }^{[9]}$ These half lives are listed in Table 2, and they range from 40 to $400 \mathrm{~h}$ indicating good $\left(4^{\circ} \mathrm{C}\right)$ storage stability. It is also clear that crosslinking the double-layer improves the stability, as reflected in both the half life and the apparent diffusivity $D_{\mathrm{e}}$ in Table 2 .

According to Table 2, the exponent $n$ decreases with increasing temperature gradually approaching 0.5 , corresponding to diffusion-controlled release (Fickian diffusion). Taking the data at $45{ }^{\circ} \mathrm{C}$ and assuming a diffusion mechanism, then the diffusion distance $(L)$ may be estimated by $L=\left(2 D_{\mathrm{e}} t\right)^{1 / 2}$ with $t$ being the reached at $45^{\circ} \mathrm{C}$ after about $300 \mathrm{~min}$. Meanwhile, the apparent diffusivity is of the order of $10^{-7} \mu \mathrm{m}^{2} \mathrm{~min}^{-1}$ according to Table 2 . This leads to an estimate of $L$ of $7.7 \mathrm{~nm}$, which seems to correspond to the single-layer thickness of F68, or the half thickness of the double-layer micelles used in these experiments. At lower temperature, the exponent $n$ rises toward one. This indicates that the diffusion of B12 across the PEO-PPO-PEO shell is either non-Fickian or there is some binding between them at the interface or inside the shell. Such behavior holds for all the F68-series nanocapsules no matter whether they are cross-linked/ IO-containing or not. This is not surprising since they all share a very similar CMT (varying from 39.3 to $40.5^{\circ} \mathrm{C}$ if we exclude unmodified $\mathrm{F} 68$, which has a CMT of $43.2^{\circ} \mathrm{C}$ ). This relatively high CMT is partially responsible for the excellent stability of the nanocapsules at room temperature and below.

\subsection{Magnetic Heating and Core Restructure}

Under magnetic heating, burst-like release was seen which is likely due to the irreversible and disruptive changes shown in diffusion time. All the data in Figure 7 suggest that $50 \%$ release is .

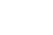


1 Figure 8 . Such changes were clearly caused by a local temperature 2 increase inside the IO, which must reach several hundred 3 degrees of centigrade since rapid diffusion in IO is not expected 4 below $300{ }^{\circ} \mathrm{C}$. This large temperature increase should not extend 5 much beyond the IO/water interface since according to the 6 standard heat conduction theory, the radial heat flux from a hot 7 sphere is inversely proportional to the radius and the temperature 8 rise is nearly completely dissipated within one radius from the sphere. This means that the temperature rise can be sustained 0 only within 2-4 $\mathrm{nm}$ from the IO/water interface, given the initial size of IO of $5-7 \mathrm{~nm}$ according to Figure $5 \mathrm{c}$ ).

In our experiment, cooling water was supplied to maintain a constant temperature in the water bath during magnetic heating. Using similar experimental configurations but without active cooling, an initial temperature rise of the water solution at a linear rate $\left(R_{\mathrm{W}}\right)$ of $0.1-1{ }^{\circ} \mathrm{C} \mathrm{s}^{-1}$ has been reported. ${ }^{[10]}$ Since the energy input to heat up the water comes entirely from the heat generated in the magnetic particles, we can calculate the heating rate $R_{\mathrm{IO}}$ of the IO nanoparticles in terms of $R_{\mathrm{W}}$ using $V_{\mathrm{IO}} R_{\mathrm{IO}}$ $20 \quad C_{\mathrm{IO}}=\left(1-V_{\mathrm{IO}}\right) R_{\mathrm{W}} C_{\mathrm{W}}$. Here $V_{\mathrm{IO}}$ is the volume fraction of IO 1 relative to the solution, and $C_{\mathrm{W}}$ and $C_{\mathrm{IO}}$ are volumetric specific 22 heat of water and IO, respectively. Our experiment and most 23 experiments in the literature used $V_{\mathrm{IO}} \sim 0.001$. Meanwhile, 24 referring to the specific heat of $\mathrm{Fe}, \mathrm{O}$, and water, we estimate $25 C_{\mathrm{W}} / C_{\mathrm{IO}} \sim 1$. Therefore, the calculated $R_{\mathrm{IO}}$ is from 100 to $1000^{\circ} \mathrm{C}$ $26 \mathrm{~s}^{-1}$, i.e., it takes at most a few seconds for the temperature to rise 27 to several hundred degrees in IO before a steady state is reached. 28 The steady-state temperature can be easily shown to scale as $G$ / $29 \alpha r^{2}$ by balancing the heat conduction (with a thermal 30 conductivity $\alpha$ ) away from IO and the heat generation rate 31 ( $G$, per unit volume) within IO, which has a radius $r$. Without 32 knowing the details of interface heat transfer at the nanoscale, 33 though, a more definitive determination of the steady-state 34 temperature is not yet possible.

\section{Conclusions}

(i) Self-assembled aqueous nanocapsules containing a hydrophilic core and a crosslinked yet temperature-sensitive shell have been successfully prepared using PEO-PPOPEO triblock copolymers, 4-nitrophenyl chloroformate
(NPC), gelatin, and 1-ethyl-3-(3-dimethylaminopropyl) car- 1 bodiimide. The core may be rendered magnetic by incorpor- 2 ating iron oxide (IO) nanoparticles that form via internal 3 precipitation.

(ii) The nancapsules are spherical exhibiting a hydrophilic-to- 6 hydrophobic transition at a characteristic but tunable 7 temperature (CMT) triggering a size contraction as much 8 as 6.5 times. With crosslinking and IO filling the size contraction decreases to a ratio of 2.6, allowing a large contraction of the core volume exceeding 17 times.

(iii) The crosslinked nanocapsules with a CMT from 39 to $43^{\circ} \mathrm{C} 13$ exhibit very little leakage at $4{ }^{\circ} \mathrm{C}$ and $25^{\circ} \mathrm{C}$ with an estimated 14 half life of 270-410 and 80-90 h, respectively, with or without 15 IO. Their half life at $37^{\circ} \mathrm{C}$ still exceeds 40 and $41.6 \mathrm{~h}$, which decreases to about $5 \mathrm{~h}$ at $45^{\circ} \mathrm{C}$ reflecting a diffusivity transition across the CMT.

(iv) Responding to external magnetic induction, magnetic nano- 20 capsules undergo irreversible structure changes including 21 IO crystal coarsening, core/shell disruption, and rapid 22 release of $80 \%$ of the core content within $5 \mathrm{~min}$. Such 23 burst-like response may be utilized for controlled drug 24 release as illustrated here using a model drug vitamin B12. 25

\section{Experimental}

Synthesis of Activated PEO-PPO-PEO Polymers (F127 and F68): We used two commercial PEO-PPO-PEO thermal-sensitive polymers, pluronic F127 and F68, (Sigma, USA) to form two series of core/shell nanocapsules in this study. F127 has a (EO) ${ }_{100}(\mathrm{PO})_{65}(\mathrm{EO})_{100}$ block structure with an EO/ $\mathrm{PO}$ ratio of 3.08 , and $\mathrm{F} 68$ has a $(\mathrm{EO})_{76}(\mathrm{PO})_{29}(\mathrm{EO})_{76}$ block structure with an $\mathrm{EO} / \mathrm{PO}$ ratio of 5.24 . The polymer ( $20 \mathrm{~g}$ of $\mathrm{F} 127$ or $\mathrm{F} 68$ ) was first dissolved in $60 \mathrm{~mL}$ of methylene chloride (Fisher Scientific, USA). In a dropwise manner this solution was added to a stirred solution of methylene chloride $(60 \mathrm{~mL})$ containing NPC $(2 \mathrm{~g})$. The activation reaction of forming covalent 37 bonds between NPC and pluronics proceeded with gentle stirring $(5 \mathrm{~h})$ at 38 room temperature under a nitrogen atmosphere. The activated polymer 39 (F127-NPC or F68-NPC) was precipitated, washed five times in ice-cold 40 diethyl ether, and dried under vacuum. To determine the activation density, 41 a known amount of F127-NPC or F68-NPC was treated with $\mathrm{NaOH}(0.2 \mathrm{~N}) \quad 42$ at $25^{\circ} \mathrm{C}(2 \mathrm{~h})$. The concentration of NPC released in the aqueous phase was 43 quantified spectrophotometrically at $410 \mathrm{~nm}$.

Fabrication of Activated PEO-PPO-PEO Nanocapsules: Single-layer 45 nanocapsules containing one layer of activated PEO-PPO-PEO were 46 synthesized using a single-emulsification (SE)/solvent-evaporation 47 method. An oil phase (methylene chloride solution, $1 \mathrm{~mL}$ ) containing 48 various amount of F127-NPC or F68-NPC (0.05-1.5 g) was added dropwise 49 to a water phase $(10 \mathrm{~mL})\left(\mathrm{W}_{2}, \mathrm{PBS}\right)$ at $\mathrm{pH}$ 7.4. The mixture was sonicated 50 (10 min) using an ultrasonic homogenizer (Virsonic, VirTis, USA) 51 operating at $20 \mathrm{kHz}$ to obtain oil-in-water micelles, F127-SE or F68-SE, 52 as shown in Figure la.

Double-layer nanocapsules containing two layers of PEO-PPO-PEO 54 were synthesized using a double-emulsification (DE)/solvent-evaporation 55 method. The first water phase $\mathrm{W}_{1}(\mathrm{PBS}, 0.1 \mathrm{~mL})$ was added dropwise to an 56 oil phase (methylene chloride solution, $1 \mathrm{ml}$ ) containing various amount of 57 F127-NPC or F68-NPC $(0.05-1.5 \mathrm{~g})$. The mixture was sonicated $(10 \mathrm{~min})$ to 58 obtain water $\left(W_{1}\right)$-in-oil micelles. Next, the micelle solution was added 59 dropwise to a second water phase $\mathrm{W}_{2}$ (PBS, $10 \mathrm{~mL}$ ). The mixture was again 60 sonicated $(10 \mathrm{~min})$ to obtain $\mathrm{W}_{1}$-in-oil-in- $\mathrm{W}_{2}$ micelles. The sample was 61 called F127-DE or F68-DE (or F127-NPC-DE and F68-NPC-DE to 62 emphasize that the outer shell is NPC-activated.) These emulsion 63 solutions were next dialyzed (dialysis membrane with an Mw cutoff of 64 14000 ) and the product stored at $4{ }^{\circ} \mathrm{C}$ until further use. The oil phase

\section{7}

28 29 30 31 32 4 6 38 1 2 4 . 48
49 53 54 .

(n)

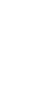

1

62
63
64

64


1 (methylene chloride) can be removed by evaporation at $30^{\circ} \mathrm{C}$ to leave a 2 double-layer micelle containing a $W_{1}$ core, yet itself suspended in the $W_{2}$ 3 phase.

Fabrication of Double-Layer Cross-linked Nanocapsules: A schematic of 5 the nanocapsules with a cross-linked shell is depicted in Figure 1a. 6 Essentially, the F127-DE or F68-DE activated with NPC is cross-linked with 7 gelatin (Sigma), a hydrolyzed natural protein polymer rich in amino and 8 carboxyl groups. To introduce gelatin to these double-layer micelles, we 9 modified the second step in their preparation and added gelatin of various 0 amount (1-10 $\mathrm{mg} \mathrm{mL}^{-1}$ ) to the $\mathrm{W}_{2}$ phase (PBS) prior to the addition of the $1 W_{1}$-in-oil micelles. The emulsion of the resultant $W_{7}$-in-oil-in- $W_{2}$ micelles 2 was immediately transferred to a water bath at $4{ }^{\circ} \mathrm{C}$ and held for $24 \mathrm{~h}$ to 3 crosslink gelatin. After that, the emulsion solution was stirred at $30^{\circ} \mathrm{C}$ to 14 remove residual methylene chloride until the solution became clear. In 15 addition, the gelatin can be fully cross-linked with the (dropwise) addition 16 of $1 \mathrm{ml}$ of $0.1 \mathrm{M}$ 1-ethyl-3-(3-dimethylaminopropyl) carbodiimide (EDC, 17 Sigma) to the above solution, held at $4{ }^{\circ} \mathrm{C}(24 \mathrm{~h})[13,14]$. These emulsion 18 solutions were also dialyzed and the product stored at $4{ }^{\circ} \mathrm{C}$ until further use. 19 The product after the first-step cross-linking will be referred to as F127-Ge 20 or $\mathrm{F} 68-\mathrm{Ge}$, and the product after the second-step cross-linking will be 21 referred to as F127-EDC or F68-EDC.

Encapsulation of Drug and Iron Oxide into Nanocapsules: The above procedure was modified to incorporate a model drug vitamin B12 (Sigma), a cobalamin, into the $\mathrm{W}_{1}$ phase; then this phase was used to prepare the double-layer nanocapsules as before followed by cross-linking. The concentration used was $10 \mathrm{mg} \mathrm{mL}^{-1}$ vitamin $\mathrm{B} 12$ in $0.1 \mathrm{~mL}$ PBS. To incorporate iron oxide, we used in situ co-precipitation of $\mathrm{Fe}$ (II) and $\mathrm{Fe}$ (III) salts. Briefly, $\mathrm{FeCl}_{3} \cdot 6 \mathrm{H}_{2} \mathrm{O}$ (Riedel-deHaën) $\left(0.1 \mathrm{~mL}\right.$ of $1.08 \mathrm{~g} \mathrm{~mL}^{-1}$ ) and $\mathrm{FeCl}_{2} \cdot 4 \mathrm{H}_{2} \mathrm{O}$ (Fluka) $\left(0.4 \mathrm{~g} \mathrm{~mL}^{-1}\right)$, together with vitamin $\mathrm{B} 12\left(10 \mathrm{mg} \mathrm{mL}^{-1}\right)$ 30 were mixed into PBS to form the $W_{1}$ phase; then this phase was used to 1 prepare the double-layer nanocapsules as before. After the final cross32 linking steps, the $\mathrm{pH}$ of the mixture solution was adjusted to 10 by adding 33 ammonia solution (33\%) under stirring, followed by heat treatment at $3460^{\circ} \mathrm{C}$ for $30 \mathrm{~min}$ to precipitate iron oxide particles, as shown in Figure $1 \mathrm{~b}$. 35 This product will be referred to as F68-IO. Other washing and storage 6 procedures are the same as before.

37 Drug Release Test: In vitro drug release from the $\mathrm{W}_{1}$ core of various 38 double-layer nanocapsules was evaluated by incubating the vitamin B1239 containing nanocapsules in $20 \mathrm{~mL}$ of PBS at various temperatures. At 40 specific time intervals, some PBS solution was withdrawn and its 41 concentration of vitamin B12 was measured by UV spectrum $(361 \mathrm{~nm})$. 42 The percentage cumulative release was determined from this concentra43 tion after normalizing it by the amount of the initially loaded vitamin B 12.

44 The data were analyzed in terms of various kinetic mechanisms by fitting it 45 to the time law of the form $[15,16]$.

$\frac{M_{t}}{M_{\infty}}=k t^{n}$ where $M_{t}$ is the cumulative release after time $t, M_{\infty}$ the cumulative release at time infinity, and $k$ and $n$ are fitting coefficients.

We attempted to increase the release rate by radio frequency magnetic heating generated by an induction heater $(15 \mathrm{~kW})$ operating at 50 $100 \mathrm{kHz} .[17,18]$ The configuration is similar to the one reported in the literature $[5,10]$, with an induction (copper) coil of eight loops delivering a magnetic field $\left(2.5 \mathrm{kA} \mathrm{m}^{-1}\right)$. The solution was kept at $15^{\circ} \mathrm{C}$ through a water bath during the experiment. The amount of the PBS solution used was $10 \mathrm{~mL}$ containing $0.2 \mathrm{~g}$ nanocapsules. Other procedures were the same as described before.
Characterization of Size and Microstructure: The chemical structure of the activated polymers was characterized by proton nuclear magnetic resonance spectroscopy ( $\left.{ }^{1} \mathrm{H}-\mathrm{NMR}\right)$ to confirm the sites and degrees of substitution. The samples were dissolved in $\mathrm{CDCl}_{3}$ and the spectra were recorded by an NMR spectrometer (Bruker Avance-500, operating at $500 \mathrm{MHz}$ ) equipped with a microprocessor-controlled gradient unit and an inverse-detection multinuclear BBI probe with an actively shielded zgradient coil. For nanocapsule characterization, dynamic light scattering (DLS, zetasizer-3000HS, Malvern, UK) was used for size determination and microscopy was performed using a transmission electron microscope (TEM, JEM-2010, JEOL, Japan) operating at $200 \mathrm{kV}$.

\section{Acknowledgements}

This work was supported by the National Science Council of the Republic of China, Taiwan under contract nos. NSC96-2627-B-009-006 and NSC962113-M009-027-MY2, and by the US National Science Foundation under grant no. DMR- 05-20020 (MRSEC). Supporting Information is available online from Wiley InterScience or from the author.

Received: September 3, 2008

Revised: November 3, 2008 Published online:

[1] J. Kost, R. Noecker, E. Kunica, R. Langer, J. Biomed. Mater. Res. 1985, 19, 935.

[2] J. Kost, J. Wolfrum, E. Kunica, R. Langer, J. Biomed. Mater. Res. 1987, 21, 1367.

[3] V. M. De Paoli, S. H. De Paoli Lacerda, L. Spinu, B. Ingber, Z. Rosenzweig, N. Rosenzweig, Langmuir 2006, 22, 5894

[4] S. H. Hu, S. Y. Chen, D. M. Liu, C. S. Hsiao, Adv. Mater. 2008, 20, 2690.

[5] A. M. Derfus, G. V. Maltzahn, T. J. Harris, T. Duza, K. S. Vecchio, E. Ruoslahti, S. N. Bhatia, Adv. Mater. 2007, 19, 3932.

[6] S. H. Choi, J. H. Lee, S. M. Choi, T. G. Park, Langmuir 2006, 22, 1758.

[7] J. B. Leach, C. E. Schmidt, Biomaterials 2005, 26, 125. References have been renumbered. Please check.

[8] L. Guo, R. H. Colby, J. Rheol. 2001, 45, 1223.

[9] S. M. Ansell, S. A. Johnstone, P. G. Tardi, L. Lo, S. Xie, Y. Shu, T. O. Harasym, N. L. Harasym, L. Williams, D. Bermudes, B. D. Liboiron, W. Saad, R. K. Prud'homme, L. D. Mayer, J. Med. Chem. 2008, 51, 3288.

[10] D. H. Kim, D. E. Nikles, D. T. Johnson, C. S. Brazel, J. Magn. Magn. Mater. 2008, 320, 2390.

[11] Z. M. Lu, D. Prouty, Z. Guo, V. O. Golub, C. S. S. R. Kumar, Y. M. Lvov, Langmuir 2005, 21, 2042.

[12] R. E. Rosensweig, J. Magn. Magn. Mater. 2002, 252, 370.

[13] W. C. Lin, T. Y. Liu, M. C. Yang, Biomaterials 2004, 25, 1947.

[14] T. Y. Liu, W. C. Lin, L. Y. Huang, S. Y. Chen, M. C. Yang, Biomaterials 2005, 25, 1437.

[15] P. L. Ritger, N. A. Peppas, J. Controlled Release 1987, 5, 37.

[16] J. S. Ahn, H. K. Choi, M. K. Chun, J. M. Ryu, J. H. Jung, Y. U. Kim, C. S. Cho, Biomaterials 2002, 23, 1411

[17] S. H. Hu, T. Y. Liu, D. M. Liu, S. Y. Chen, Macromolecules 2007, 40, 6786.

[18] S. H. Hu, T. Y. Liu, H. Y. Huang, D. M. Liu, S. Y. Chen, Langmuir 2008, 24, 239.

Q1: Author: Please clarify throughout the article all editorial/

*: Author: Please note that Figure of Toc will be printed in colour. technical requests marked by black boxes. 